\title{
Acute pancreatitis caused by cytomegalovirus-associated duodenal papillitis
}

\author{
Yuko Sakakibaraa, Shoichi Nakazurua, Yoshinori Kodama ${ }^{b}$, Eiji Mitaa
}

National Hospital Organization, Osaka National Hospital, Osaka, Japan

A 66-year-old man presented to our hospital with fever and epigastralgia. He was treated with oral steroid therapy for 2 months prior to admission for renal failure caused by antineutrophil cytoplasmic antibody-associated vasculitis. Laboratory data showed elevated levels of amylase (499 IU/L; normal, 42-132 IU/L) and lipase (272 IU/L; normal, 13-49 IU/L). Computed tomography (CT) and magnetic resonance cholangiopancreatography showed enlargement of the head of the pancreas and dilatation of the main pancreatic duct (MPD). Esophagogastroduodenoscopy (EGD) showed erythematous and edematous major papilla (Fig. 1). A histopathological examination of biopsy specimens, taken from the major papilla, revealed active duodenitis, with a cytomegalovirus (CMV) infection confirmed by immunostaining (Fig. 2A). An examination for CMV antigenemia (C7-HRP) showed 63 positive cells per 50,000 cells (Fig. 2B). Based on these findings, the papillitis was determined to be caused by the CMV infection. After 1 month of treatment with intravenous ganciclovir, a CT scan showed that the dilatation of the MPD and enlargement of the head of the pancreas had improved; in addition, a repeat EGD indicated improvement in the edematous major papilla. Since then, symptoms related to pancreatitis have not recurred. Although CMV is a common opportunistic pathogen infecting patients taking immunosuppressive drugs, CMV-associated duodenal papillitis is rare [1-3]. The possibility of acute pancreatitis caused by $\mathrm{CMV}$-associated duodenal papillitis should be considered among immunosuppressed patients.

Departments of a Gastroenterology and Hepatology (Yuko Sakakibara,

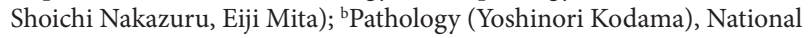
Hospital Organization, Osaka National Hospital, Osaka, Japan

Correspondence to: Yuko Sakakibara, MD, Department of Gastroenterology and Hepatology, National Hospital Organization, Osaka National Hospital, 2-1-14 Houenzaka, Chuo-ku, Osaka city, Osaka 540-0006, Japan, e-mail: yuko.s@onh.go.jp

Conflict of Interest: None

Received 3 August 2017; accepted 7 August 2017; published online 6 September 2017

DOI: https://doi.org/10.20524/aog.2017.0188

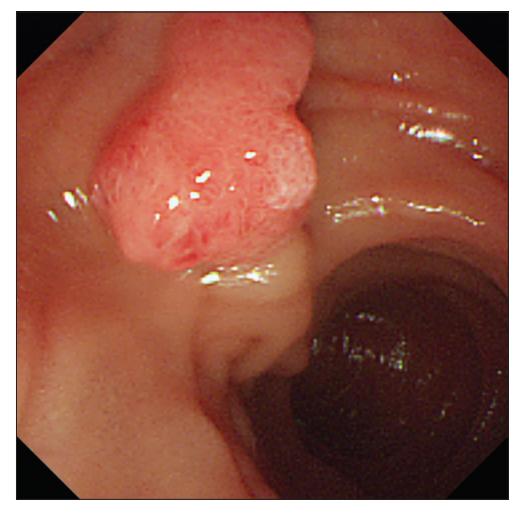

Figure 1 Endoscopic image showing an edematous major papilla

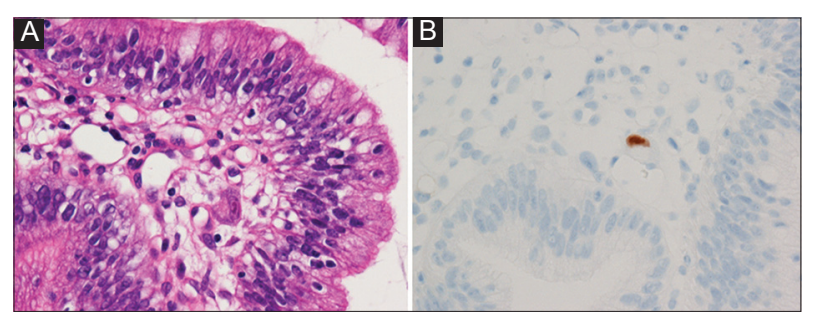

Figure 2 Photomicrographs of the biopsy specimen taken from the major papilla. (A) A very small number of cytomegalovirus inclusions were seen (hematoxylin and eosin stain; original magnification, 400×). (B) The cells with cytomegalovirus inclusions showing positive immunoreactions to the presence of cytomegalovirus (original magnification, $400 \times$ )

\section{References}

1. Kim YS, Cho YD, Lee JS, Jin SY, Shim CS. Cytomegalovirus infection in an HIV patient with duodenal papillitis. Endoscopy 2007;39(Suppl 1):E23.

2. Kamalkumar BS, Agarwal SK, Garg P, Dinda A, Tiwari SC. Acute pancreatitis with CMV papillitis and cholangiopathy in a renal transplant recipient. Clin Exp Nephrol 2009;13:389-391.

3. Riediger C, Beimler J, Weitz J, et al. Cytomegalovirus infection of major duodenal papilla in a renal allograft recipient with severe biliary obstruction and acalculous cholecystitis. Transpl Infect Dis 2013;15:E129-E133. 